\title{
QUANTITATIVE SURFACE EMISSIVITY AND TEMPERATURE MEASUREMENTS OF A BURNING SOLID FUEL ACCOMPANIED BY SOOT FORMATION
}

\author{
Nancy D. Piltch, NASA Glenn Research Center, Cleveland, Ohio, \\ Richard D. Pettegrew, NCMR, Member AIAA, and \\ Paul Ferkul, NCMR, Senior Member AIAA
}

\section{Abstract}

Surface radiometry is an established technique for noncontact temperature measurement of solids. We adapt this technique to the study of solid surface combustion where the solid fuel undergoes physical and chemical changes as pyrolysis proceeds, and additionally may produce soot. The physical and chemical changes alter the fuel surface emissivity, and soot contributes to the infrared signature in the same spectral band as the signal of interest. We have developed a measurement that isolates the fuel's surface emissions in the presence of soot, and determine the surface emissivity as a function of temperature. A commercially available infrared camera images the two-dimensional surface of ashless filter paper burning in concurrent flow. The camera is sensitive in the 2 to $5 \mu \mathrm{m}$ band, but spectrally filtered to reduce the interference from hot gas phase combustion products. Results show a strong functional dependence of emissivity on temperature, attributed to the combined effects of thermal and oxidative processes. Using the measured emissivity, radiance measurements from several burning samples were corrected for the presence of soot and for changes in emissivity, to yield quantitative surface temperature measurements. Ultimately the results will be used to develop a full-field, non-contact temperature measurement that will be used in spacebased combustion investigations.

Copyright 82001 by the American Institute of Aeronautics and Astronautics, Inc. No copyright is asserted in the United States under Title 17, U.S. Code. The U.S. government has a royalty-free license to exercise all rights under the copyright claimed herein for Governmental purposes. All other rights are reserved by the copyright owner.
Introduction

Infrared (IR) thermography as a technique for noncontact temperature field measurements of solid surfaces requires knowledge of the emissive properties of the surface for accurate interpretation of the IR signal. In general, the surface emissivity and other radiative parameters are a function of temperature, wavelength, and viewing angle, as well as surface composition and finish $^{1,2,3}$. While the radiative properties of various materials have been examined under limited conditions $^{4,5,6,7,8}$, materials undergoing the physical and chemical changes of combustion introduce additional complexity. In particular, emissions from the flame must be isolated, for example by optical filtering, from the broadband surface radiation. Soot, if present, further complicates the interpretation of the signal, as it is a broadband emitter, and therefore cannot be removed by filtering. Finally, in the mid-IR region where our detector operates, there is only a small spectral window that is free of gas phase emission, so that twowavelength pyrometry is not feasible.

This paper describes a technique for the experimental determination of the temperature dependence of the emissivity of a burning thin solid fuel in the presence of soot. Part of this technique is a procedure to reduce the effect of soot on the temperature field measurements for moderately sooty flames. As two-dimensional temperature field measurements are ultimately desired, all measurements were made in an imaging configuration.

\section{$\underline{\text { Formulation }}$}

The measurement scenario consists of imaging a burning sample surface with a mid-IR camera, while simultaneously acquiring an independent point temperature measurement on the sample surface, using a thermocouple. The image is filtered to block gas-phase emissions (from $\mathrm{CO}, \mathrm{CO}_{2}, \mathrm{H}_{2} \mathrm{O}$ and vapor phase hydrocarbons). A small hole in the fuel sample, near the thermocouple, allows the imaging of soot (if present) from

1 American Institute of Aeronquitics and Astronautics conference. Because changes may be made before formal publication, this is made available with the understanding that it will not be cited or reproduced without the permission of the author. 
both sides of the sample. The signal from the soot can be subtracted from the detector signal, leaving only the IR emissions of the solid surface. The corrected detector signal and the thermocouple measurement together provide the surface emissivity. The correction reduces to the familiar ratio of actual radiance to blackbody radiance when soot is absent.

Figure 1 shows a schematic layout of the fuel surface.

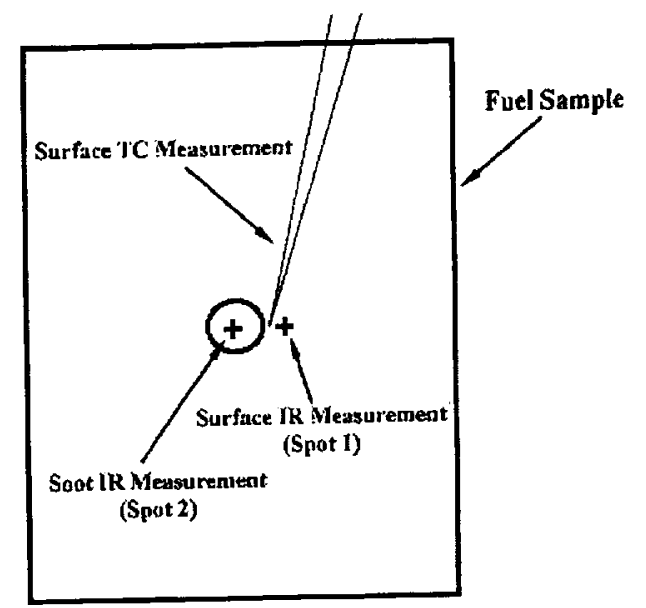

Figure 1

\section{Fuel Surface Arrangement and Measurement Locations}

A Type $\mathrm{K}$ thermocouple mounted to the fuel surface with a bit of epoxy provided an independent temperature measurement, needed for the determination of surface emissivity. Once the emissivity was determined, the thermocouple was no longer required and the measurement scheme becomes truly non-contact.

The contribution of soot to the total radiance was isolated by simultaneously imaging through a small hole in the fuel. Imaged radiance there includes only emissions from soot in flames on both sides of the burning fuel. The hole diameter was chosen to be much larger than the spatial resolution of the camera, so that a local soot radiation measurement could be made without also collecting radiation from the nearby sample surface. The hole was located along the centerline of the fuel, and the thermocouple was mounted on the surface nearby along a horizontal line

The measurement requires the following assumptions: (i) the fuel surface and soot emissions, the bandpass (flame reject) filter transmission and the detector sensitivity are spectrally uniform within the filter bandpass, (ii) lateral temperature variations (perpendicular to the flame spread direction) are small, (iii) scattering and absorption of radiation by soot can be neglected, (iv) the detector response is approximately linear and, (v) the imaging depth of field is sufficiently large to include the flames on both sides of the burning sample.

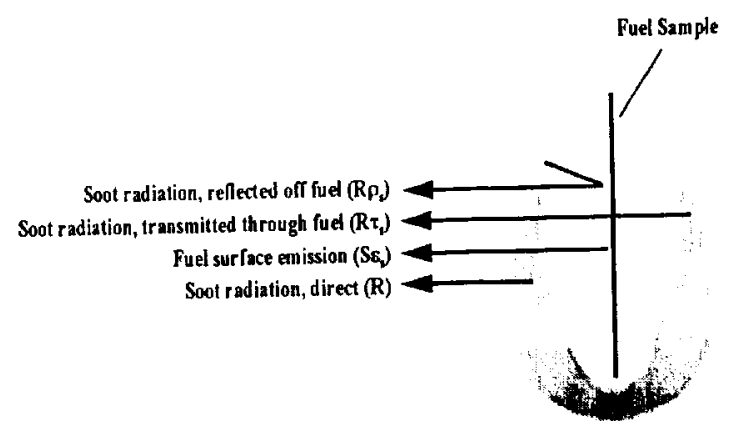

Figure 2

\section{Radiance Components in the Observed Image}

Figure 2 shows a schematic of the radiant contributions to the detector signal. The total radiance arriving at the detector from spot 1 in figure $1, R_{T O T}$, is:

$$
R_{\text {TOT }}=\rho_{s} R+\tau_{s} R+\varepsilon_{s} S_{B}+R
$$

Where the soot radiance from one side of the flame is $R$, the blackbody radiance from the surface is $S_{B}$, and the radiative properties of the fuel are $\varepsilon_{s}, \tau_{s}$, and $\rho_{s}$ for the emissivity, transmissivity and reflectivity.

The time-encoded data were the total front view radiance coming from the fuel surface and soot (Eq. 1), the radiance of the soot alone, measured through the hole in the fuel surface, and the thermocouple output. All radiance signals were collected in detector counts, proportional to photons received. The thermocouple signal was converted to equivalent counts by calibration against a blackbody source. Thermocouple data were not corrected for radiative losses. The detector signal for spot 1 on the fuel surface is $S_{1}$, and represents $R_{T o r}$; the signal from spot 2 is $S_{2}$. Since the flame is symmetric front and back, $S_{2}$ is twice the value of the singleside soot radiance $R$, where scattering and selfabsorption are neglected. No assumption of soot emis- 
sivity is required. Apart from a proportionality constant representing collection geometry, camera throughput, and $\mathrm{A} D \mathrm{D}$ conversion factors,

$$
\begin{aligned}
& S_{1}=R_{\mathrm{TOT}} \\
& S_{2}=2 \mathrm{R}
\end{aligned}
$$

Conservation of energy gives:

$$
1=\varepsilon_{s}+\rho_{s}+\tau_{s}
$$

Substituting and rearranging gives:

$$
\varepsilon_{\mathrm{s}}=\left(\mathrm{S}_{1}-\mathrm{S}_{2}\right) /\left(\mathrm{S}_{\mathrm{B}}-\mathrm{S}_{2} / 2\right)
$$

Note that we treat $\rho_{\mathrm{s}}+\tau_{\mathrm{s}}$ as a single variable and do not determine the two quantities independently.

\section{Experimental Setup}

The fuel used in this work was Whatman \#4 Ashless Filter Paper, a thin, cellulosic material similar to other fuels used in combustion studies ${ }^{9,10}$. The $5 \times 10 \mathrm{~cm}$ sample was taped to a thin metal holder and placed in a $26-$ liter chamber. A $0.27 \mathrm{~mm}$ diameter (29 gauge) resistively heated Kanthal wire, woven in a sawtooth pattern on the bottom edge of the fuel, provided the ignition source resulting in upward flame spread. A Type $\mathrm{K}$ thermocouple (3-mil wire diameter) mounted about 5 $\mathrm{cm}$ up from the ignited edge, just to the side of the centerline, using a dot of epoxy for good thermal contact provided the independent surface temperature measurement. At the centerline of the sample (next to the thermocouple) a 5-mm hole allowed imaging of the soot field alone. The spatial resolution of the camera (at $30 \mathrm{~cm}$ working distance using a 20-degree forelens with close-up attachment) is approximately $0.3 \mathrm{~mm}$, so that a $5 \mathrm{~mm}$ diameter hole allowed such a measurement. Images were recorded in the mid-IR using a commercially available camera (single element detector, scanning type) with a $3.8+1-0.05 \mu \mathrm{m}$ bandpass filter which effectively eliminated contributions from $\mathrm{CO}, \mathrm{CO}_{2}$, $\mathrm{H}_{2} \mathrm{O}$ and vapor phase hydrocarbons. The detector dynamic range permitted imaging over a temperature range of approximately $400 \mathrm{~K}$ to $750 \mathrm{~K}$, which corresponds to preheat and pyrolysis of the fuel surface. Images were recorded at $30 \mathrm{~Hz}$ video frame rate and matched up with nearly simultaneous thermocouple data. All tests were conducted in normal gravity, with $21 \% \mathrm{O}_{2}$ at a pressure of $28 \mathrm{kPa}$.

\section{Experimental Results}

Experimental results showing the surface emissivity (in the 3.7-3.9 $\mu \mathrm{m}$ band) as a function of temperature are plotted in Figure 3. This figure shows eight tests that followed a similar qualitative trend, although with sig-

\section{Emissivity vs. TC Temperature (K)}

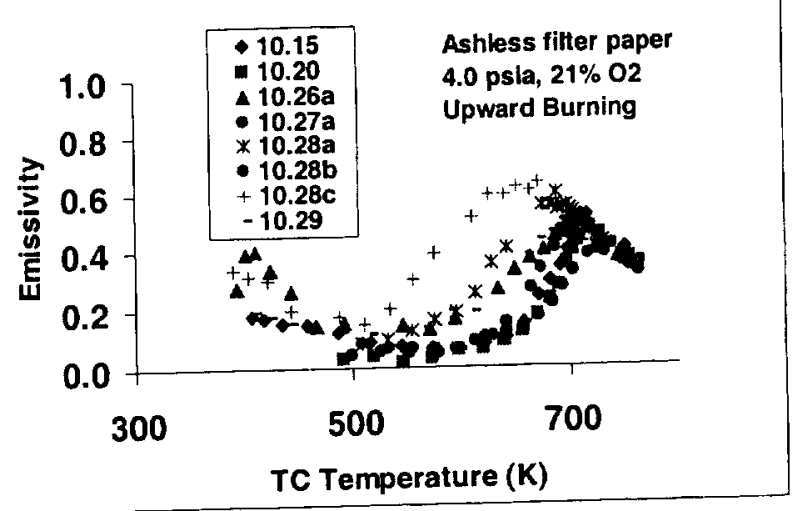

Figure 3

\section{Surface Emissivity in the 3.7-3.9um Band, as a Function of Temperature}

nificant scatter. These tests indicate a value of $\varepsilon_{s}(T)$ of approximately $0.3+/-0.1$ at the lowest recorded temperatures of about $400 \mathrm{~K}$. The emissivity then drops to a minimum of about 0.1 near $530 \mathrm{~K}$, climbs to a maximum of about 0.6 by $680 \mathrm{~K}$, and finally drops sharply to slightly below 0.4 by $750 \mathrm{~K}$, at which point the tests terminated as the fuel was consumed.

These eight tests are averaged to determine a relation between emissivity and temperature for the fuel used here. Using this relation, we can calculate the fuel surface temperature using only the two IR signal measurements $\left(S_{1}\right.$ and $S_{2}$, described earlier).

Figure 4 shows the surface temperature (at a point) calculated in this manner for two tests, along with the actual thermocouple traces. The calculated curves do not replicate the thermocouple traces exactly, due to the averaging of the emissivity over multiple tests. These differences (between the calculated IR temperature and the recorded thermocouple temperature) are an indication of the limitation of this technique, which depends on how well the emissivity-temperature curve is known. 


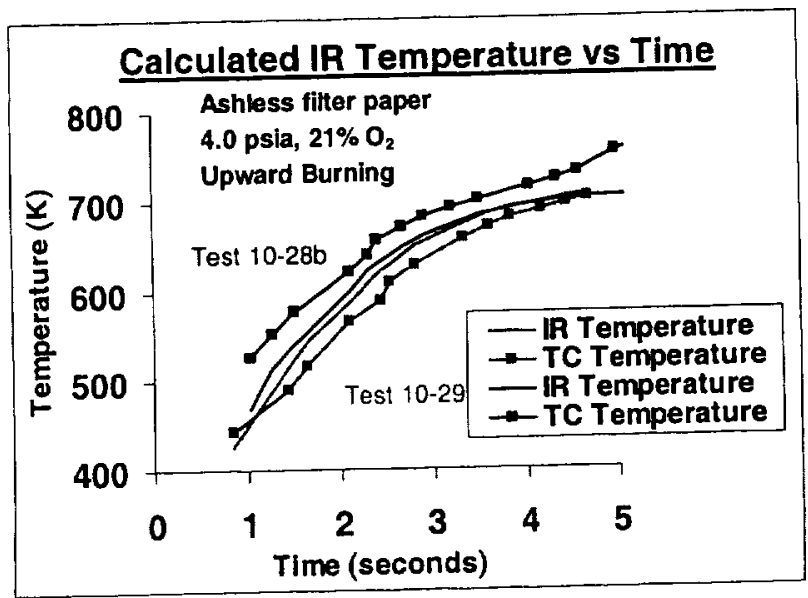

Figure 4

Calculated IR Temperature and Thermocouple Temperature vs. Time (including soot correction)

\section{Discussion}

These tests show a low overall emissivity and a strong temperature dependence of the emissivity. Therefore, constant emissivity values reported in various references ${ }^{11,12}$ may not be appropriate for burning samples. The reported measurements may have been made at a different wavelength, different temperature, or in nonpyrolyzing environments. It is not intuitive that the value of the emissivity should be as low as that determined here; the literature ${ }^{11,12}$ states that the emissivity of paper should be in the range of 0.8 to 1.0. A plausible explanation for this discrepancy can be seen by recalling that the current measurements were made in a narrow spectral bandpass $(3.7-3.9 \mu \mathrm{m})$. Figure 5 shows the mid-infrared absorption spectrum of Kimwipes (trade name) a cellulosic laboratory wipe frequently used in combustion studies at room temperature. While this spectrum changes with temperature, and may differ in detail from that for ashless filter paper, it demonstrates that the radiative properties can be very different in a narrow bandpass compared to the overall spectrally averaged quantity. The spectral region that is free of gas phase emission is a region of relatively low absorbance and therefore low emissivity. The same spectral region will be used for the noncontact field thermometry, which is the ultimate goal of the project and therefore is the region in which these calibrations must be performed.
The non-monotonic thermal behavior of the emissivity may be somewhat surprising. Though not yet confirmed, this behavior is believed to be a result of the combined effects of both pyrolysis and oxidation leading to structural and chemical changes in the fuel. There appears to be a qualitatively similar result reported for cellulose undergoing pyrolysis without combustion ${ }^{13}$. Emissivity for this case was measured by FTIR reflectance assuming zero transmittance. Our values differ numerically, due to the narrow bandpass of our measurement and our inclusion of transmittance.

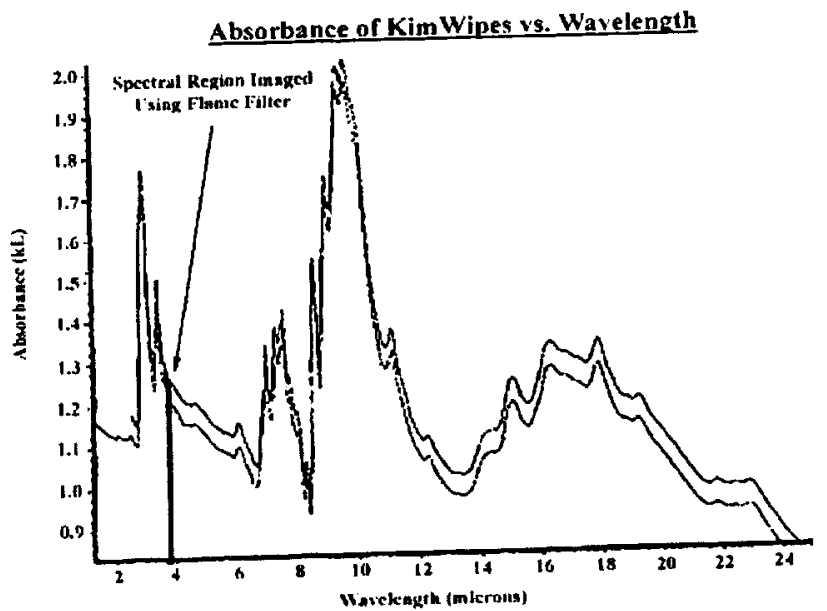

Figure 5

Spectrally Dependent Absorbance of KimWipes at Room Temperature, Using an FTIR

An area of concern is the validity of neglecting scatter and self-absorption of radiation by soot. This is equivalent to the standard simplified models of gas emission. The simplified model is expected to capture most of the observed phenomena with greater accuracy than complete neglect of soot effects. With improved knowledge of soot distribution in these flames, a more complete approach may be developed if the measurement precision warrants it.

The influence of soot on the observed radiance is shown in Figure 6. This figure shows the measured emissivity of the fuel as a function of temperature, and was one of the test points used in this study. The upper trace is calculated from the raw data without correcting for the presence of soot, while the lower trace includes our correction. The greatest difference is at low temperatures, corresponding to the preheat region. In this

4 


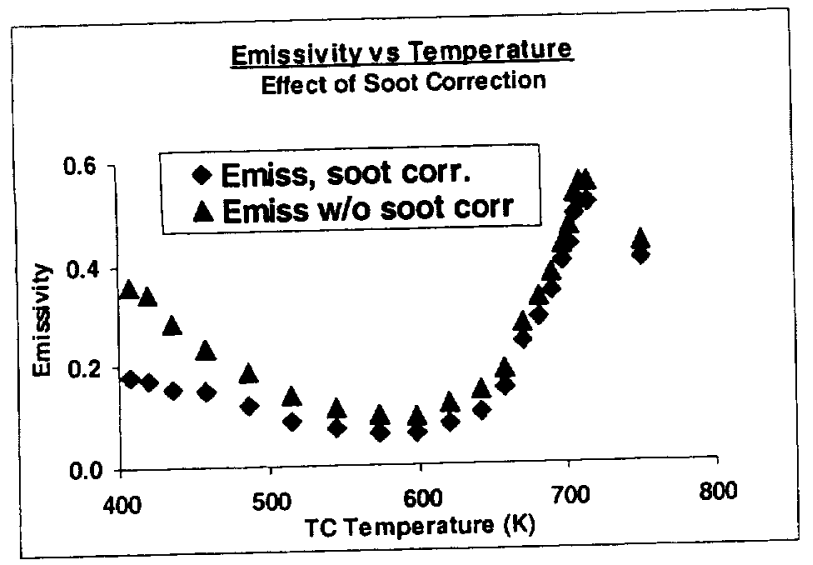

Figure 6

\section{Surface Emissivity (3.7-3.9um Band) as a Function of Temperature, with and without Soot Correction}

region, the downstream tip of the flame, which has the highest soot volume fraction, appears in the field of view of the camera and contributes substantial radiance to the observed signal. As the flame propagates downstream, the fuel surface temperature rises and its radiance increases. Simultaneously, the camera views a less sooty portion of the flame, so that soot contributes proportionately less radiance. The two traces are essentially identical within experimental error for temperatures higher than about $550 \mathrm{~K}$.

The high amount of test-to-test scatter in the emissivity measurements requires some comment. One of the factors that contributes to the experimental scatter is the mounting technique of the thermocouples. The junctions were fixed to the sample surface using epoxy; however, the exact amount of epoxy used varied slightly from test to test. The size of the thermocouple beads in the various tests (along with the epoxy used to mount them) was approximately 0.22 to $1.5 \mathrm{~mm}$. The corresponding thermal response times reach a maximum of about $0.5 \mathrm{msec}$, indicating that the thermal inertia of the bead is not a concern.

The thermal length scale of the flame $\left(\alpha / \mathrm{V}_{\text {buoyant }}\right)$ is approximately $1 \mathrm{~mm}$. Hence, a temperature gradient may occur across the bead, introducing an uncertainty in the temperature measurement. Since the determination of the emissivity is based on correlation between the thermocouple data and IR data, the uncertainty in the thermocouple data will propagate into the emissiv- ity results. Additional scatter may arise from spot-tospot variation in the surface condition.

The theoretical limit to which the emissivity can be determined (using the equipment in this study, based on the manufacturers' stated detector and thermocouple accuracies) is estimated to be $\pm 12 \%$ (relative error). This translates to a maximum uncertainty in surface temperature of $\pm 15 \mathrm{~K}$. Clearly we have not yet reached this limit, but the technique described here has the potential for improvement when the independent surface temperature measurement (thermocouple) is refined. We will attempt this in future work.

\section{Conclusions}

Tests of the technique were conducted using thin paper fuel (ashless filter paper) in upward burning normal gravity tests. These tests show a strong dependence of emissivity on temperature, with values everywhere much lower than those commonly published. The low values are attributed to the low spectral absorption by cellulose in the narrow band used to eliminate interference by gas phase emissions. These effects must be accounted for in any experimental effort that uses IR thermography for quantitative temperature measurement.

Examination of the data from these tests shows a fair amount of scatter; much of this is thought to be due to inconsistencies with the mounting technique of the surface thermocouples. The uncertainties caused by this effect are thought to account for the variations observed from test to test.

The measurements show a significant influence of soot radiance on the determination of- surface emissivity, particularly in the downstream (preheat) region. The effect of the soot-signal compensation algorithm was studied by calculating the surface emissivity while first including, then neglecting soot. The test data indicate that for the conditions and configuration employed, failure to account for soot can cause over prediction of the emissivity in this region. For the particular case studied, the over prediction was about a factor of two.

In summary, a technique was developed for determining the temperature-dependent emissivity of a thin burning sample. This method provides a simple way to isolate emissions from the soot field in the gas phase flame and implicitly includes the effects of physical and chemical changes occurring in the fuel surface during 
combustion. The technique is applicable to a wide range of materials and up to moderate soot loading. No assumption of optical opacity of the fuel is required. Ultimately it may permit non-contact thermometry without the need for a surface-mounted thermocouple and without the reliance on tabulated emissivity values generated under different conditions.

\section{Acknowledgements}

The authors wish to express their gratitude to the NASA Aeronautics and Space Administrations' Office of Biological and Physical Research who supported this work.

Trade names or manufacturers' names are used in this report for identification only. This usage does not constitute an official endorsement, either expressed or implied, by the National Aeronautics and Space Administration.

${ }^{1}$ Siegel, R., and Howell, J.R.: Thermal Radiation Heat Transfer, Hemisphere Publishing Corporation, Washington (1981).

${ }^{2}$ Arakawa, A., Saito, K., and Gruver, W.A.: Automated Infrared Imaging Temperature Measurement with Application to Upward Flame Spread Studies, Part I, Combustion and Flame, Vol. 92, pp. 222-230 (1993).

${ }^{3}$ Staaf, O., Ribbing, C.G., and Andersson, S.K.: Temperature Dependence of the Band Emittance for Non-gray Bodies, Applied Optics, Vol. 35, No. 31, pp. 6120-6125 (1996).

${ }^{4}$ Haugh, M.J.: Infrared Thermometry for Low Emissivity Metals, Instrumentation Society of America Transactions, Vol. 22, No. 3 (1983).

${ }^{5}$ Olstad, S.J., Tanaka, F., and DeWitt, D.P.: Evaluation of a Method for Measuring Spectral Emissivity at Moderate Temperatures, AIAA $20^{\text {th }}$ Thermophysics Conference, AIAA-85-0991 (1985).

${ }^{6}$ Zhang, Y.W., Zhang, and C.G., Klemas, V.: Quantitative Measurements of Ambient Radiation, Emissivity, and Truth Temperature of a Greybody: Methods and Experimental Results, Applied Optics, Vol. 25, No. 20, pp.3683-3689 (1986).
${ }^{7}$ Pettegrew, R., Piltch, N., and Ferkul, P.: Emissivity Measurement of a Thin, Pyrolyzing Solid, $37^{\text {th }}$ AIAA Aerospace Sciences Conference, AIAA 99 0700 (1999)

${ }^{8}$ Serio, M.A.., Pines, D.S., Bonano, A.S., Solomon, P.R.: An Instrument for Characterization of the Thermal and Optical Properties of Charring Polymeric Materials, pp. 1447-1453, 25 th $^{\text {Symposium (International) }}$ on Combustion, The Combustion Institute, 1994

${ }^{9}$ Bhattacharjee, S., Altenkirch, R.A., Olson, S.L., Sotos, R.G.: Heat Transfer to a Thin Solid Combustible in Flame Spreading at Microgravity, Journal of Heat Transfer-Transactions of the ASME, 113:(3) 670676, August, 1991

${ }^{10}$ Bhattacharjee, S., Altenkirch, R.A, Sacksteder, K.: Effect of Ambient Pressure on Flame Spread Over a Thin Cellulosic Fuel in a Quiescent, Microgravity Environment, Journal of Heat TransferTransactions of the ASME, 118:(1) 181-190, February, 1996

${ }^{11}$ Grober, H., Erk, S., and Grigull, U.: Fundamentals of Heat Transfer, $3^{\text {rd }}$ Edition, McGraw-Hill Series in Mechanical Engineering, (1961)

${ }^{12}$ Incropera, F.P., and DeWitt, D.P.: Fundamentals of Heat and Mass Transfer, 3rd Edition, John Wiley\& Sons, (1981).

${ }^{13}$ Suuberg, E.M., Milosavljevic, I., and Lilly, W.D.: Behavior of Charring Materials in Simulated Fire Environments, NIST-GCR-94-645 (1994). 